\title{
Seroprevalence of Neutralizing Antibodies to Human Adenovirus Type 4 and 7 in Healthy Populations From Southern China
}

\section{OPEN ACCESS}

Edited by:

Qiwei Zhang,

Southern Medical University, China

Reviewed by:

Adriana E. Kajon,

Lovelace Respiratory Research

Institute, United States

Wenbo Xu,

Chinese Center for Disease Control

and Prevention, China

Gabriel Gonzalez,

Hokkaido University, Japan

*Correspondence:

Ling Chen

chen_ling@gibh.ac.cn

Liqiang Feng

feng_liqiang@gibh.ac.cn

${ }^{\dagger}$ These authors have contributed equally to this work

Specialty section:

This article was submitted to

Virology,

a section of the journal

Frontiers in Microbiology

Received: 02 July 2018 Accepted: 26 November 2018 Published: 10 December 2018

Citation:

Ye X, Xiao L, Zheng $X$, Wang J, Shu T, Feng Y, Liu X, Su W, Wang $Q$, Li C, Chen L and Feng L (2018)

Seroprevalence of Neutralizing Antibodies to Human Adenovirus Type 4 and 7 in Healthy Populations

From Southern China.

Front. Microbiol. 9:3040.

doi: 10.3389/fmicb.2018.03040

\begin{abstract}
Xianmiao Ye ${ }^{1,2 t}$, Lijun Xiao ${ }^{3 t}$, Xuehua Zheng ${ }^{1 t}$, Jinlin Wang ${ }^{1,2}$, Tao Shu',2, Ying Feng ${ }^{4}$, Xinglong Liu', Wan Su1,5, Qian Wang ${ }^{1,2}$, Chufang Li ${ }^{4}$, Ling Chen ${ }^{1,4 *}$ and Liqiang Feng ${ }^{1 *}$

${ }^{1}$ State Key Laboratories of Respiratory Diseases, Guangzhou Institutes of Biomedicine and Health, Chinese Academy of Sciences, Guangzhou, China, ${ }^{2}$ University of Chinese Academy of Sciences, Beijing, China, ${ }^{3}$ Center for Disease Control and Prevention of Chenzhou, Chenzhou, China, ${ }^{4}$ The First Affiliated Hospital of Guangzhou Medical University, Guangzhou, China, ${ }^{5}$ School of Biomedical Sciences, Huaqiao University, Quanzhou, China
\end{abstract}

Human adenoviruses type 4 (HAdV4) and 7 (HAdV7) are two major respiratory pathogens and sporadically cause outbreaks of acute respiratory diseases. The neutralizing antibody $(\mathrm{nAb})$ response to these two adenoviruses in civilian populations, which is important for dissecting previous circulations and predicting potential outbreaks, remains largely unknown. In this study, we generated replication-competent HAdV4 and HAdV7 reporter viruses expressing secreted-alkaline-phosphatase (SEAP), and established neutralization assays to investigate the seroprevalence of pre-existing $\mathrm{nAb}$ in healthy volunteers from Hunan Province, southern China. The seropositivity rates are 58.4 and $63.8 \%$ for anti-HAdV4 nAb and anti-HAdV7 nAb, respectively. High nAb titers (> 1000) were frequently detected in HAdV4-seropositive individuals, whereas most HAdV7-seropositive volunteers had moderate nAb titers (201-1000). The seropositivity rates of anti-HAdV4 nAb and anti-HAdV7 nAb increase with age, with individuals younger than 20 exhibiting the lowest seropositivity rates. Both seropositivity rates and nAb titers are comparable between different sex groups. Notably, HAdV4seropositive individuals tend to be HAdV7-seropositive and vice versa. Because HAdV4 antisera showed no neutralizing activity to HAdV7 whereas HAdV7 antisera cannot neutralize HAdV4, a subgroup of individuals might be susceptible to infection by HAdV4 and HAdV7 and thus generate nAb to both of them. These results revealed the continuous circulation of HAdV4 and HAdV7 and the lack of protective immunity in more than $35 \%$ of people, which emphasized the surveillance of these two HAdVs and the development of prophylactic vaccines.

Keywords: human adenovirus type 4, human adenovirus type 7, neutralizing antibody, seroprevalence, Southern China

\section{INTRODUCTION}

Human adenoviruses (HAdVs) are non-enveloped, double-stranded DNA viruses belonging to Adenoviridae. To date, more than 85 genotypes of HAdVs have been identified and classified into seven species (A-G) on the basis of biological characteristics and genomic homology (Kajan et al., 2017; Hashimoto et al., 2018). HAdVs are common pathogens responsible for a variety of diseases, 
including acute respiratory disease (ARD), conjunctivitis, gastroenteritis, urinary infection and meningoencephalitis (Ghebremedhin, 2014; Radke and Cook, 2018). HAdV infection in immuno-competent healthy adults is usually asymptomatic (Berciaud et al., 2012). However, in particular populations such as immuno-compromised individuals, infants, and children, HAdV infections may lead to severe and even fatal diseases (Ghebremedhin, 2014; Radke and Cook, 2018). Outbreaks of HAdV infection are frequently reported in crowded populations such as school students and military recruits (Ghebremedhin, 2014).

HAdV4 and HAdV7, two members belonging to species $\mathrm{E}$ and $B$ respectively, are major causative pathogens of ARD and acute conjunctivitis (Hong et al., 2001; Erdin et al., 2015). Outbreaks of ARD or conjunctivitis caused by HAdV4 have been reported in the United States, Australia, and Japan (McNeill et al., 2000; Aoki and Tagawa, 2002; Zhang et al., 2016), whereas HAdV7-associated epidemics have also been documented in the United States, China, Japan and the Philippines (Tang et al., 2011; Yamamoto et al., 2014; Scott et al., 2016; Yang et al., 2017). These endemics and epidemics have rendered significant economic and healthy burdens in both military and civilian populations (Hoke and Snyder , 2013). The application of live-oral vaccines against HAdV4 and HAdV7 greatly reduced HAdV-associated ARDs in United States military trainees (Hoke and Snyder , 2013). During the cessation of vaccination between 1999 and 2011, HAdV infections sharply increased to pre-vaccine level (Erdman et al., 2002; Kajon et al., 2007). A retrospective survey revealed that low level of neutralizing antibody (nAb) to HAdV4 and HAdV7 in unimmunized military recruits facilitated the return of $\mathrm{HAdV}$ infections (Barraza et al., 1999). Although the new live-oral HAdV4 and HAdV7 vaccines were approved and re-introduced in 2011, vaccination was restricted to military recruits but was not applicable for general populations due to safety concerns (Radin et al., 2014; Choudhry et al., 2016). There is still no adenovirus vaccine for civilian populations worldwide. Besides, the knowledge about the seroprevalence of anti-HAdV4 $\mathrm{nAb}$ and anti-HAdV7 nAb in civilian populations is quite limited. We proposed that an investigation of pre-existing $\mathrm{nAb}$ could help to understand previous circulations of these two HAdVs and to predict potential outbreaks.

Recently, replication competent and incompetent genetic vectors based on HAdV4 and HAdV7 have been developed (Weaver, 2014; Peng et al., 2016). These vectors have great potency for oral administration (Deal et al., 2013). Several vaccine candidates against human immunodeficiency virus, hepatitis $B$ virus, respiratory syncytial virus, and influenza A H5N1 virus based on HAdV4 or HAdV7 vectors have been undergoing preclinical and clinical trials, and exhibited good safety and immunogenicity (Hung et al., 1990; Hsu et al., 1992; Alexander et al., 2012; Weaver, 2014; Khurana et al., 2015). However, preexisting $\mathrm{nAb}$ to HAdV4 and HAdV7 may limit the efficacy of these vaccines (Dharmapuri et al., 2009; Lanzi et al., 2011). Therefore, investigating the seroprevalence of anti-HAdV4 $\mathrm{nAb}$ and anti-HAdV7 nAb can also help to select subpopulations suitable for the application of these vaccines.
In this study, we constructed replication-competent HAdV4 and HAdV7 expressing secreted-alkaline-phosphatase (SEAP), and established convenient neutralization assays based on these reporter viruses. Using these neutralization assays, we investigated the seroprevalence of anti-HAdV4 $\mathrm{nAb}$ and antiHAdV7 $\mathrm{nAb}$ in a civilian cohort from Hunan Province, southern China. We also analyzed the distributions of seropositivity rates and $\mathrm{nAb}$ titers in different age and sex groups.

\section{MATERIALS AND METHODS}

\section{Recombinant HAdV4 and HAdV7 Reporter Viruses}

HAdV4 (GenBank NO. KF006344.1) and HAdV7 (GenBank No. HQ659699), two clinical isolates, were kindly provided by Prof. Rong Zhou (Guangzhou Medical University). Recombinant HAdV4 and HAdV7 expressing SEAP were constructed according to previously described methods (Zheng et al., 2017). In brief, viral genomic DNA was extracted by sodium-dodecylsulfonate lysis (Sigma-Aldrich, St Louis, MO, United States) followed by phenol-chloroform extraction. The terminal regions of HAdV4 and HAdV7 genome, which were used as homology arms, were amplified by PCR and subcloned into pMD19T-Simple vectors (TaKaRa, Dalian, China) to obtain shuttle plasmids pT-Ad4 $(\mathrm{L}+\mathrm{R})$ and $\mathrm{pT}-\mathrm{Ad} 7(\mathrm{~L}+\mathrm{R})$, respectively. A unique restriction site for BamHI was introduced between the two arms, and two restriction sites for AsiSI were introduced at the $5^{\prime}$ terminal of the left arm and the $3^{\prime}$ terminal of the right arm. After digestion with BamHI (Thermo Fisher Scientific, Waltham, MA, United States), linearized pT-Ad4 $(\mathrm{L}+\mathrm{R})$ and pT-Ad7 $(\mathrm{L}+\mathrm{R})$ were subjected to homologous recombination with viral genomes in E. coli BJ5183 competent cells (Agilent Technologies, Santa Clara, CA, United States) to obtain genomic plasmids pAd4 and pAd7, respectively. Subsequently, the homology arms for E3 deletion, which contain 500 base pairs (bp) upstream of HAdV4 E3 region, or 520 bp upstream of HAdV7 E3 region, or 710 bp downstream of HAdV4 E3 region, or 660 bp downstream of HAdV7 E3 region, were amplified by PCR. Two shuttle plasmids, p4E3LR and p7E3LR, were constructed by inserting the respective homology arms into pVAX1 plasmids (Thermo Fisher Scientific, Waltham, MA, United States). The ligated homology arms were released from p4E3LR and p7E3LR by digestion with BstZ17I and SgrAI (Thermo Fisher Scientific, Waltham, MA, United States). Meanwhile, pAd4 and pAd7 were linearized by digestion with BclI and EcoRI (Thermo Fisher Scientific, Waltham, MA, United States), respectively. The E3 regions were then deleted by homologous recombination between E3LR fragments and linearized pAd4 and pAd7 to obtain pAd4 $\triangle \mathrm{E} 3$ and pAd7 $\triangle \mathrm{E} 3$, respectively. To construct recombinant HAdV4 and HAdV7 reporter viruses, the coding sequences for SEAP flanked by a CMV promoter and a $\mathrm{BGH}$ poly(A) signal were amplified by PCR using pGA1-SEAP as the template (Zheng et al., 2017), and inserted into p4E3LR and p7E3LR to obtain shuttle reporter plasmids pGK43-SEAP and pGK73-SAEP, respectively. Finally, pGK43-SEAP and pGK73-SAEP were linearized by digestion with BstZ17I and SgrAI, whereas genomic plasmids 
pAd4 $\Delta \mathrm{E} 3$ or pAd7 $\Delta \mathrm{E} 3$ were linearized by digestion with SwaI (Thermo Fisher Scientific, Waltham, MA, United States), and pAd4-SEAP and pAd7-SEAP were constructed by homologous recombination between linearized shuttle reporter plasmids and genomic plasmids. To rescue HAdV4-SEAP and HAdV7-SEAP, pAd4-SEAP and pAd7-SEAP were linearized by digestion with AsiSI (Thermo Fisher Scientific, Waltham, MA, United States) and transfected into HEK293 cells (ATCC). After successfully rescued, HAdV4-SEAP and HAdV7-SEAP were propagated in HEK293 cells and purified using cesium chloride gradient centrifugation. The infectious virus titers were determined as described previously (Aste-Amezaga et al., 2004).

\section{Human Serum Samples}

A total of 1302 healthy blood donors were included in this study. The serum samples were collected in the medical examination center of Center for Disease Control and Prevention of Chenzhou, Hunan Province, China, from January 1, 2017 to July 20, 2017. The volunteers ranged from 7 to 70 years old. The percentages of male and female were 54.1 and $45.9 \%$, respectively. The demographic information of serum samples was summarized in Supplementary Table S1. Fresh blood samples were collected into serum tubes and let stand for $30 \mathrm{~min}$ at room temperature. Serum samples were then isolated by centrifugation at $3000 \mathrm{rpm}$ for $10 \mathrm{~min}$. 1302 serum samples were obtained, heat-inactivated for $90 \mathrm{~min}$ at $56^{\circ} \mathrm{C}$ and preserved at $-80^{\circ} \mathrm{C}$. The research with human samples was approved by the Ethics Committee of Guangzhou Institutes of Biomedical and Health $(\mathrm{GIBH})$. Informed consents from each volunteer were signed.

\section{Mouse Antisera}

Six-week-old female BALB/c mice were purchased from Beijing Vital River Laboratory Animal Technology Co., Ltd., and housed in the Animal Experimental Center of GIBH. Animal experiments were approved by Institutional Animal Care and Use Committee of GIBH (IACUC, No. 2015014). HAdV4 antisera and HAdV7 antisera were prepared according to previously described methods (Feng et al., 2018). In brief, HAdV4 and HAdV7 were treated with $0.2 \%$ of $\beta$-propiolactone (BPL) (Tokyo Chemical Industry, Japan) overnight at $4^{\circ} \mathrm{C}$ followed by incubation for $2 \mathrm{~h}$ at $37^{\circ} \mathrm{C}$. In this condition, HAdVs were completely inactivated. Mice were then intramuscularly inoculated with inactivated HAdV4 or HAdV7 at $1.0 \times 10^{9}$ viral particles (vp) per mouse. At 4 weeks after the first immunization, the mice were boosted with similar formulations. At 2 weeks post the final immunization, the mice were bled and euthanized. Serum samples were then collected, inactivated and preserved at $-80^{\circ} \mathrm{C}$.

\section{HAdV4 and HAdV7 Neutralization Assays}

The $\mathrm{nAb}$ titers against HAdV4 and HAdV7 were determined using neutralization assays based on HAdV4-SEAP and HAdV7SEAP, respectively. In brief, HEK293 cells were seeded into 96-well plates at $3 \times 10^{4}$ cells per well. One day later, HAdV4SEAP or HAdV7-SEAP at $4 \times 10^{6} \mathrm{vp}$ per well $(\sim 100 \mathrm{vp} /$ cell $)$ were incubated for $1 \mathrm{~h}$ at $37^{\circ} \mathrm{C}$ either alone (virus infection alone) or together with serial dilutions of human sera or mouse antisera. Subsequently, each sample was added to the 96-well plates and incubated for $24 \mathrm{~h}$ at $37^{\circ} \mathrm{C}$. Finally, the supernatants were harvested and the SEAP activity was assessed using the chemiluminescent substrate CSPD according to the manufacturer's protocols (Thermo Fisher Scientific, Waltham, MA, United States). The relative light units (RLU) were recorded using a luminometer (MlX Microtiter, Dynex Technologies, Inc., United States) and the titers were calculated as the dilutions that inhibited $50 \%$ of RLU values.

\section{Statistical Analysis}

Comparisons of the seropositivity rates in different groups were conducted using Chi-square test or Chi-square test for trend. Comparisons of the $\mathrm{nAb}$ titers among groups were performed using the Mann-Whitney test or the Kruskal-Wallis test. SPSS version 13.0 (SPSS Inc., Chicago, IL) was used for statistical analyses, and $p$-values less than 0.05 were considered statistically significant. The graphs were generated with GraphPad Prism version 6 (GraphPad Software, La Jolla, CA, United States).

\section{RESULTS}

\section{Establishment of Neutralization Assays With HAdV4-SEAP and HAdV7-SEAP}

To establish a new neutralization assay alternative for traditional cytopathic effect based neutralization assays, we constructed recombinant $\mathrm{HAdV} 4$ and $\mathrm{HAdV} 7$ reporter viruses expressing SEAP (Figure 1A). HAdV4-SEAP and HAdV7-SEAP were successfully rescued and propagated. The SEAP activity was linearly correlated with the infection dosage of HAdV4-SEAP and HAdV7-SEAP from $0.01 \mathrm{vp} /$ cell to $100 \mathrm{vp} /$ cell (Figure 1B). We chose an infection dosage of $100 \mathrm{vp} /$ cell for assessing the titers of $\mathrm{nAb}$ in the serum samples.

We established the neutralization assays using mice antisera generated by immunizing BALB/c mice with inactivated HAdV4 or HAdV7 viral particles. The neutralization curve of antisera to HAdV4-SEAP and HAdV7-SEAP were shown (Figures 1C,D). SEAP activity in the culture supernatants sharply decreased at certain dilutions of serum samples, reflecting the neutralization of antisera to HAdV4 and HAdV7. Notably, SEAP expression in cells infected with HAdV4-SEAP was only suppressed by HAdV4 antisera but not significantly influenced by HAdV7 antisera, whereas SEAP expression in HAdV7-SEAP infected cells was only inhibited by HAdV7 antisera but not HAdV4 antisera. This result suggested that HAdV4 specific nAb have minimal, if any, cross-neutralizing activity to $\mathrm{HAdV} 7$, and vice versa.

\section{Overall Seroprevalence of Anti-HAdV4 $n A b$ and Anti-HAdV7 nAb in Healthy Populations From Hunan Province, Southern China}

Using reporter virus based neutralization assays, we investigated the seroprevalence of anti-HAdV4 $\mathrm{nAb}$ and anti-HAdV7 nAb in a cohort of 1302 healthy volunteers from Human Province, China (Supplementary Table S1). The overall seropositivity 
A

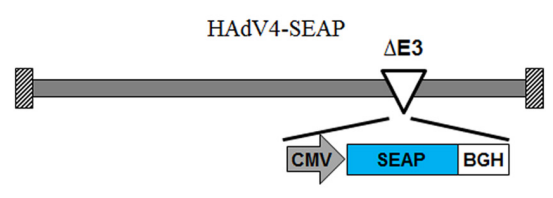

HAdV7-SEAP

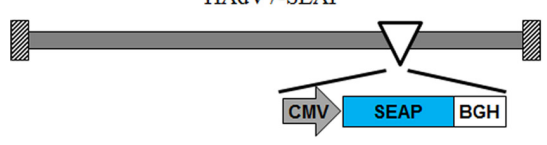

C

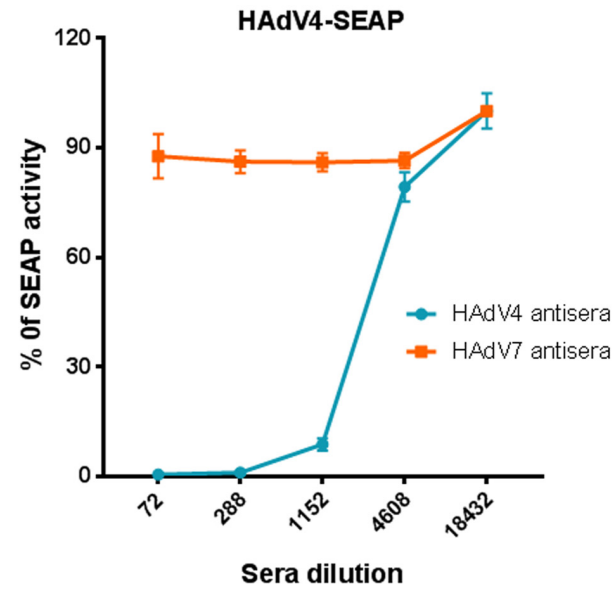

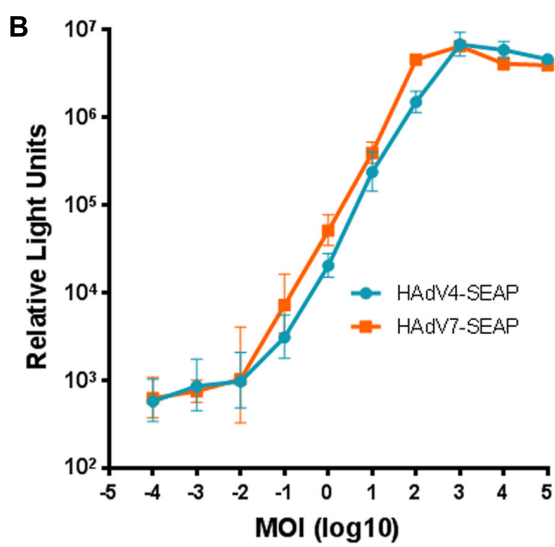

D

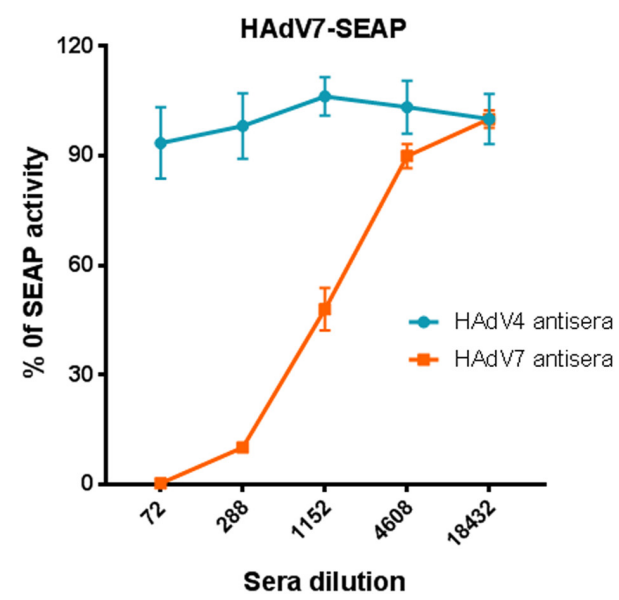

FIGURE 1 | Establishment of neutralization assays based on HAdV4-SEAP and HAdV7-SEAP. (A) The schematic diagram of HAdV4-SEAP and HAdV7-SEAP. (B) Detection of SEAP activity in the culture supernatants of HAdV4-SEAP or HAdV7-SEAP infected cells. HEK293 cells were infected with HAdV4-SEAP or HAdV7-SEAP at serially increasing doses. At $24 \mathrm{~h}$ after infection, the culture supernatants were collected and the SEAP activity was assessed. Shown are the representative data of three independent experiments. (C,D) Neutralization curves of HAdV4 antisera and HAdV7 antisera to HAdV4-SEAP (C) and HAdV7-SEAP (D). Mouse antisera were serially diluted and incubated with HAdV4-SEAP (C) or HAdV7-SEAP (D). After incubation for 1 h, the mixtures were added to HEK293 cells. One day later, the SEAP activity in the culture supernatants were examined. Cells infected with HAdV4-SEAP or HAdV7-SEAP in the absence of antisera were used as viral infection alone controls. Shown are the percentages of SEAP activity in the presence of antisera versus viral infection alone. The data were representative of two independent experiments. Error bars stand for mean \pm standard deviation (SD).

rate of anti-HAdV4 $\mathrm{nAb}$ was 58.4\% (95\% Confidence Interval, CI, 55.7-61.1\%), which was significantly lower than that of anti-HAdV7 nAb (63.8\%, 95\% CI 61.2-66.4\%) (Chi-square test, $P<0.01$; Supplementary Tables S2, S3). In the whole cohort, HAdV4-seropositive individuals had significantly higher $\mathrm{nAb}$ titers than HAdV7-seropositive ones (Mann-Whitney test, $P<0.001$, Figure 2A). According to nAb titers, the serum samples were categorized into four subgroups: negative, $<72$; low, 72-200; moderate, 201-1000; and high, $>1000$. The distributions of different $\mathrm{nAb}$ titers were analyzed (Figure 2B). $34.0 \%$ of serum samples (95\% CI 32.6-38.0\%) contained high nAb titers to HAdV4, whereas only $22.9 \%$ (95\% CI 21.9-26.9\%) of serum samples had high nAb titers to HAdV7 (Chi-square test, $P<0.001)$. Significantly more serum samples contained moderate nAb titers to HAdV7 (26.8\%; 95\% CI 24.4-29.2\%) than to HAdV4 (16.3\%; 95\% CI 14.3-18.3\%) (Chi-square test, $P<0.001$, Figure 2B). These results suggested that although the seropositivity rate of anti-HAdV4 $\mathrm{nAb}$ was lower than that of anti-HAdV7 nAb, HAdV4-seropositive individuals tended to have higher $\mathrm{nAb}$ titers in comparison with HAdV7-seropositive individuals.

\section{Seropositivity Rates but Not Titers of Anti-HAdV4 nAb and Anti-HAdV7 nAb Increased With Age}

We analyzed the trends of seroprevalence in different age groups. The overall seropositivity rates of anti-HAdV4 $\mathrm{nAb}$ and antiHAdV7 nAb increased with age (Chi-square test for trend, HAdV4: $P<0.001$; HAdV7: $P<0.001$; Figure 3A). The frequency of serum samples with moderate and high anti-HAdV4 $\mathrm{nAb}$ and anti-HAdV7 nAb titers also progressively increased with age (Figures 3B,C). However, the frequency of serum samples with low anti-HAdV4 $\mathrm{nAb}$ and anti-HAdV7 nAb titers increased 

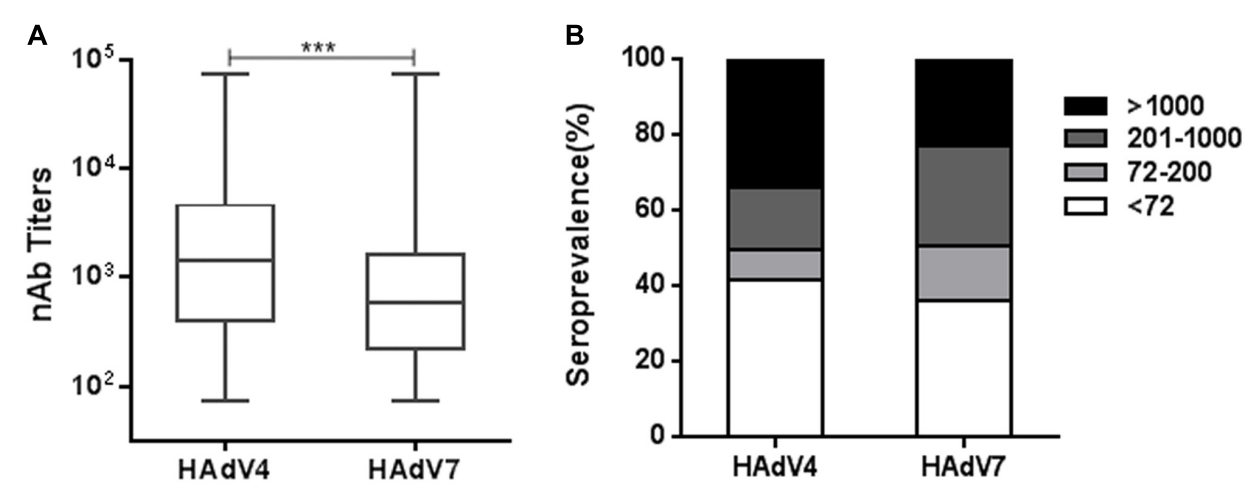

FIGURE 2 | The overall seroprevalence of anti-HAdV4 nAb and anti-HAdV7 nAb. (A) The nAb titers against HAdV4 and HAdV7 in seropositive individuals. The data was analyzed with the Mann-Whitney test. ${ }^{* * *} P<0.001$. (B) The distributions of anti-HAdV4 nAb and anti-HAdV7 nAb titers. nAb titers were classified into four subgroups: negative, < 72; low, 72-200; moderate, 201-1000; and high, > 1000. The percentages of individuals with negative, low, moderate, and high nAb titers were shown.

with age before 50 but decreased thereafter (Figures 3B,C). Notably, in each age group, the percentage of individuals with high anti-HAdV4 $\mathrm{nAb}$ titers was higher than that with moderate and low anti-HAdV4 nAb titers (Figure 3B), whereas individuals with moderate anti-HAdV7 nAb titers dominated in HAdV7seropositive ones (Figure 3C). The titers of anti-HAdV4 nAb was higher in the age group younger than 20 than the other age groups (Kruskal-Wallis test, $P<0.001$; Figure 3D). However, no difference was observed for the titers of anti-HAdV7 nAbs among age groups (Kruskal-Wallis test, $P=0.399$; Figure 3E). These results revealed a different distribution of seropositivity rates as well as nAb titers for HAdV4 and HAdV7, and implied that individuals younger than 20 may be more susceptible to HAdV4 and HAdV7 infection than older individuals because of relatively lower $\mathrm{nAb}$ seropositivity rates.

\section{Seropositivity Rates and Titers of Anti-HAdV4 nAb and Anti-HAdV7 nAb Were Not Affected by Sex}

We next analyzed the seroprevalence and titer distributions of anti-HAdV4 $\mathrm{nAb}$ and anti-HAdV7 $\mathrm{nAb}$ in sex groups. The seropositivity rates of anti-HAdV4 $\mathrm{nAb}$ in males was slightly higher than that in females, but this difference did not reach statistical significance (Figure 4A). Similarly, no significant differences were observed for the seroprevalence of anti-HAdV7 nAb between males and females (Figure 4B). The titers of anti-HAdV4 $\mathrm{nAb}$ and anti-HAdV7 $\mathrm{nAb}$ in seropositive males were also comparable to those in seronegative females (Figures 4C,D). Therefore, the seroprevalence of anti-HAdV4 $\mathrm{nAb}$ and anti-HAdV7 nAb were associated with age, but not with sex.

\section{Analysis of the Seroprevalence and nAb Titers in Double-Positive and Single-Positive Individuals}

We also analyzed the seropositivity rates of anti-HAdV4 nAb in HAdV7-seropositive or -seronegative individuals. Significantly more HAdV4-seropositive samples were detected in HAdV7seropositive individuals than in HAdV7-seronegative ones (Chisquare test, $P<0.001$; Figure 5A). The percentages of sera with low, moderate and high anti-HAdV4 nAb titers in HAdV7seropositive individuals were similar to those in HAdV7seronegative ones (Figure 5B). Similarly, the seropositivity rate of anti-HAdV7 $\mathrm{nAb}$ in HAdV4-seropositive individuals was significantly higher than that in HAdV4-seronegative ones (chi-square test, $P<0.001$; Figure 5A). The percentages of sera with low, moderate and high anti-HAdV7 nAb titers in HAdV4-seropositive individuals were also comparable to those in HAdV4-seronegative ones (Figure 5C). Moreover, the titers of anti-HAdV4 $\mathrm{nAb}$ and anti-HAdV7 $\mathrm{nAb}$ in double-positive sera were comparable to that in single-positive sera (Figures 5D,E). These results revealed that HAdV7-seropositive individuals tend to be HAdV4-seropositive, and vice versa, but the nAb titers to these two HAdVs were comparable in single- and double-positive individuals.

\section{DISCUSSION}

HAdV4 and HAdV7 represent two major respiratory pathogens in civilian populations and military trainees worldwide (Top, 1975; Tang et al., 2011; Yamamoto et al., 2014). However, surveillances of $\mathrm{nAb}$ to these two HAdVs in large civilian cohorts are extremely rare, possibly due to the lack of convenient neutralization assays. Here, we developed neutralization assays using HAdV4-SEAP and HAdV7-SEAP (Figure 1). These assays have been shown to be more sensitive, convenient, and objective than traditional cytopathic effect based methods (AsteAmezaga et al., 2004; Zheng et al., 2017), and thus enabled the surveillance of pre-exiting anti-HAdV4 $\mathrm{nAb}$ and anti-HAdV7 $\mathrm{nAb}$ in large cohorts. To our knowledge, we are the first to report the seroprevalence of $\mathrm{nAb}$ to HAdV4 and HAdV7 in civilian population. We found that, the seropositivity rates of anti-HAdV4 $\mathrm{nAb}$ and anti-HAdV7 $\mathrm{nAb}$ were 58.4 and $63.8 \%$ (Figure 2), respectively, which were lower than that of antiHAdV5 nAb but higher than that of anti-HAdV14 nAb and 


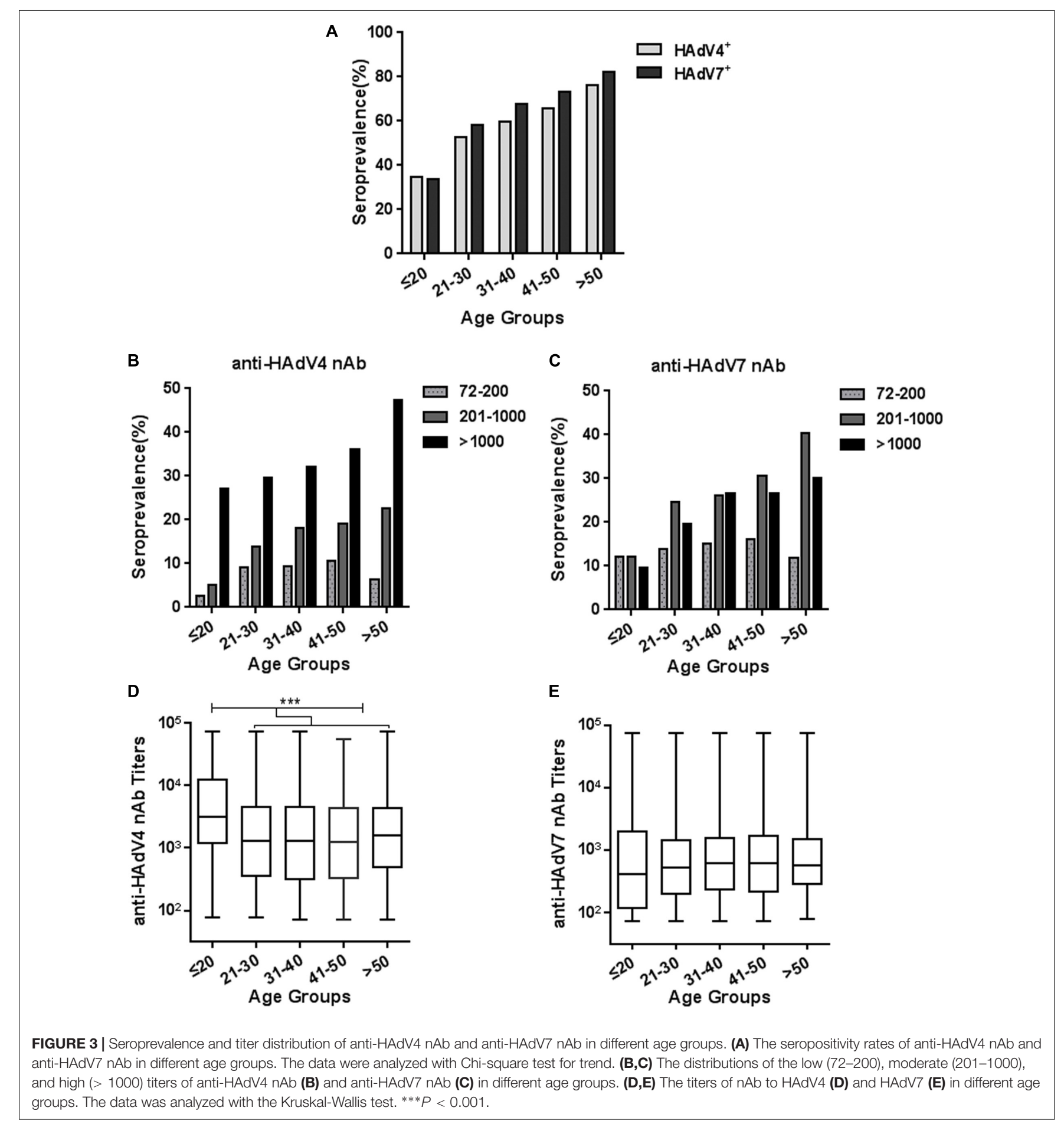

anti-HAdV55 nAb (Hong et al., 2001; Sun et al., 2011; Zheng et al., 2017). At least 41.6 and $36.2 \%$ of individuals in this population are still susceptible for HAdV4 and HAdV7 infection respectively, due to the absence of protective $\mathrm{nAb}$ (Barraza et al., 1999; Clemmons et al., 2017). Our results reinforced that HAdV4 and HAdV7 should be considered when ARD outbreaks. A prophylactic vaccine may be necessary for the prevention of HAdV4 and HAdV7 infection.
Recently, the pre-existing $\mathrm{nAb}$ to a variety of HAdVs including HAdV2, 5, 14, 26, 35, and 55 have been investigated (Barouch et al., 2004; Abbink et al., 2007; Zheng et al., 2017). However, Investigations of anti-HAdV4 $\mathrm{nAb}$ and anti-HAdV7 $\mathrm{nAb}$ were mainly conducted in military recruits (Ludwig et al., 1998; Kolavic-Gray et al., 2002; Choudhry et al., 2016). Results from United States military trainees revealed that the overall seroprevalence of anti-HAdV4 nAb was significantly lower than 

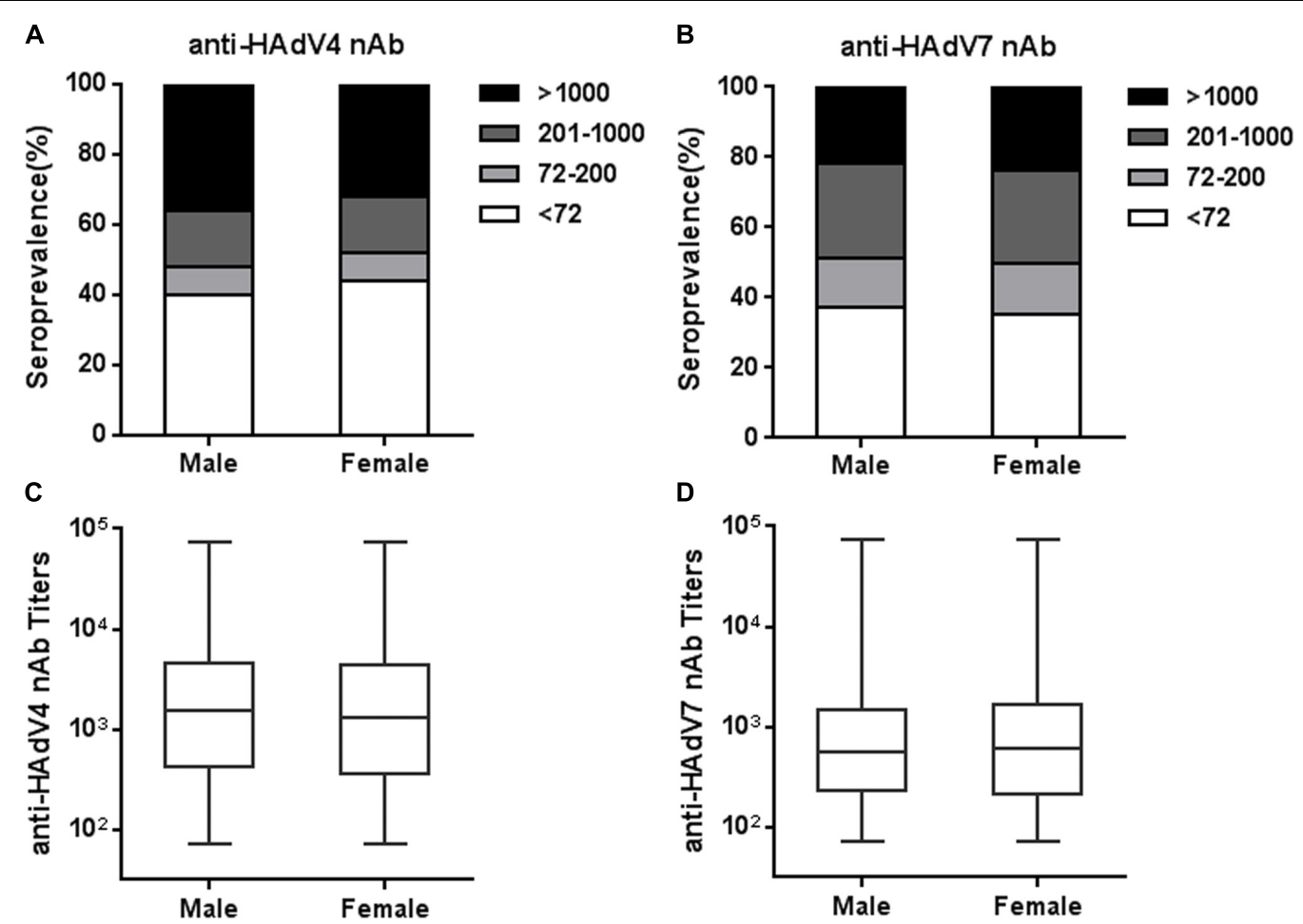

FIGURE 4 | Seroprevalence and titer distribution of anti-HAdV4 nAb and anti-HAdV7 nAb in sex groups. (A,B) The distributions of anti-HAdV4 nAb (A) and anti-HAdV7 nAb (B) titers in sex groups. (C,D) The titers of nAb to HAdV4 (C) and HAdV7 (D) in seropositive males and females. The data were analyzed using the Mann-Whitney test.

that of anti-HAdV7 nAb, whereas individuals with high titers of anti-HAdV4 nAb were much more than those with high titers of anti-HAdV7 nAb (Ludwig et al., 1998). Our results in civilian populations showed a similar trend. That is, the frequency of HAdV4-seropositive individuals is lower than that of HAdV7seropositive ones, but the titers of anti-HAdV4 nAb were significantly higher than that of anti-HAdV7 nAb (Figure 2). The similar results from two different populations imply that HAdV4 circulation in these populations may be more recent than HAdV7, or HAdV4 infection may induce stronger $\mathrm{nAb}$ responses than HAdV7 infection.

Several studies have shown that the seroprevalence of $\mathrm{nAb}$ to other HAdVs such as HAdV5 increased with age (Sun et al., 2011; Yu et al., 2012), but the nAb titers would decrease gradually in years post infection due to cumulative saturation of infection in adults (Yang et al., 2016). However, we found that both HAdV4 and HAdV7 seropositivity rates gradually increased with age (Figure 3), consistent to previous results from army trainees (Ludwig et al., 1998), suggesting that infection rates of HAdV4 and HAdV7 would not be saturated in adults. The sporadic appearance of adult patients with HAdV4- or HAdV7associated severe pneumonia also supported the absence of protective immunity in a proportion of individuals (Barraza et al., 1999; Clemmons et al., 2017). Thus, close attention should be paid to HAdV4- and HAdV7-associated ARDs not only for children, but also for adults. Similar to HAdV14 and HAdV55 seroprevalence (Zheng et al., 2017), sex is not an important factor influencing either seropositivity rates or nAb titers of HAdV4 and HAdV7 (Figure 4). Intriguingly, we found that HAdV4seropositive individuals tend to be HAdV7-seropositive, and vice versa (Figure 5). Given that HAdV4 antisera and HAdV7 antisera showed no significant cross-neutralizing activity to HAdV4 and HAdV7 (Figure 1), this result implied that a proportion of individuals might be more susceptible to HAdV infection. These individuals may be successively or simultaneously infected by HAdV4 and HAdV7 and thereby generated $\mathrm{nAb}$ responses to both of them. However, the mechanisms underlying this phenomenon need to be elucidated in future studies.

Recombinant adenoviral vectors based on HAdV4 and HAdV7 have been explored as vaccine vectors for a variety of pathogenic viruses (Hung et al., 1990; Hsu et al., 1992; Alexander et al., 2012; Weaver, 2014; Khurana et al., 2015). A potential limitation of recombinant adenoviral vectors is the pre-existing $\mathrm{nAb}$ resulted from natural exposure (Lanzi et al., 2011). The sensitive neutralization assays established in this study can potentially facilitate the screening of HAdV4- and HAdV7seronegative individuals, which may be important for the clinical evaluation of vaccines based on recombinant HAdV4 or HAdV7 vectors. In this particular cohort, 41.6 and $36.2 \%$ of participants were seronegative for anti-HAdV4 $\mathrm{nAb}$ and anti-HAdV7 nAb, respectively, and thereby could be included in the clinical trials for HAdV4- or HAdV7-vectored vaccines.

HAdV4 has been shown to originate from a recombinant event between HAdV16 and simian adenovirus type 26 (SAdV26) 


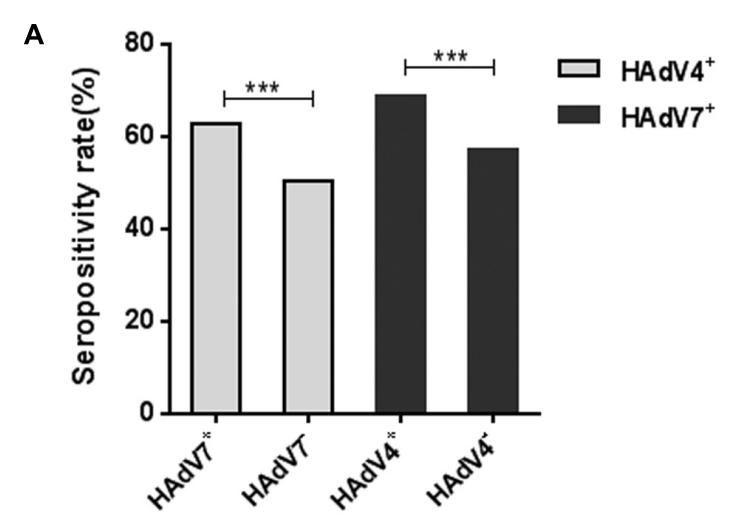

B

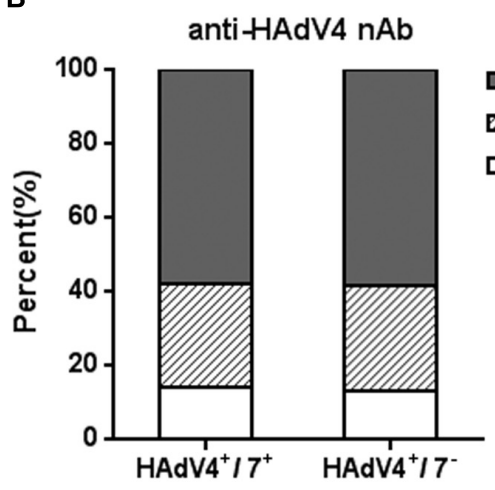

D

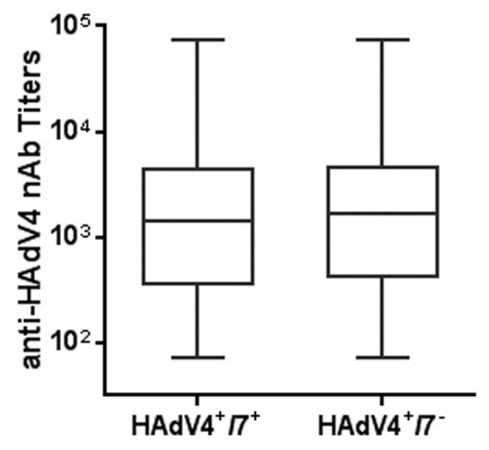

C

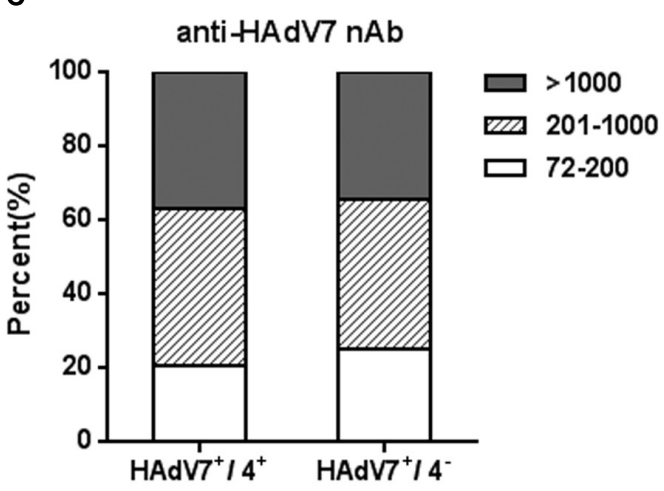

E

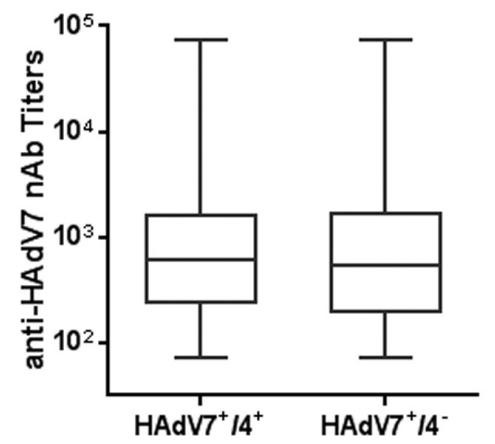

FIGURE 5 | Seropositivity rates and titers of anti-HAdV4 nAb and anti-HAdV7 nAbs in single- and double-positive samples. (A) The seropositivity rates of anti-HAdV4 nAb and anti-HAdV7 nAb in single-positive and double-positive serum samples. The data were analyzed with Chi-square test. ${ }^{* * *} P<0.001$. (B,C) The distributions of anti-HAdV4 nAb (B) and anti-HAdV7 nAb (C) titers in single-positive and double-positive serum samples. (D,E) Overall titers of anti-HAdV4 nAb (D) and anti-HAdV7 nAb (E) in single-positive and double-positive serum samples. The data were analyzed using the Mann-Whitney test.

(Dehghan et al., 2013), and comprises loop 1 and loop 2 of HAdV16 hexon in the genome chassis of SAdV26. Because hexon loop 1 and loop2 contain the major targets of anti-HAdV nAb (Pichla-Gollon et al., 2007; Yuan et al., 2009; Feng et al., 2018), screening of anti-HAdV4 nAb with HAdV4-SEAP cannot discriminate the $\mathrm{nAb}$ induced by HAdV16 infection. However, dissecting the seroprevalence of anti-HAdV4 $\mathrm{nAb}$, resulted no matter from HAdV4 infection or from HAdV16 infection, could help to understand the herd immunity to HAdV4. Moreover, to our best knowledge, no outbreaks of HAdV16 infection have ever been reported in Hunan province, China. The circulation of HAdV16 in this district is not dominant and even rare. Therefore, the pre-existing anti-HAdV4 $\mathrm{nAb}$ in the investigated cohorts was most likely resulted from HAdV4 infection but not HAdV16 infection. Future studies, such as detection of fiber-specific nAb and surveillance of the circulating HAdV strains may help to clarify the real seroprevalence of anti-HAdV4 $\mathrm{nAb}$ induced by HAdV4 infection.

In summary, we developed reporter virus based neutralization assays for HAdV4 and HAdV7, and investigated the pre-existing $\mathrm{nAb}$ responses in a civilian population. HAdV4 and HAdV7 can be potential causative pathogens for ARDs in civilian populations in southern China. Our results provided insightful information not only for understanding the herd immunity to these two 
HAdVs but also for the development of vaccines against HAdV infection.

\section{DATA AVAILABILITY STATEMENT}

The raw data supporting the conclusions of this manuscript will be made available by the authors, without undue reservation, to any qualified researcher.

\section{AUTHOR CONTRIBUTIONS}

$\mathrm{XY}$, LX, and XZ collected the samples, performed the neutralization assays and analyzed the results. JW, TS, YF, XL, WS, and QW contributed to the data analysis. CL reviewed the manuscript. LC and LF conceived and designed the study, wrote the manuscript, and approved the submission.

\section{REFERENCES}

Abbink, P., Lemckert, A. A., Ewald, B. A., Lynch, D. M., Denholtz, M., Smits, S., et al. (2007). Comparative seroprevalence and immunogenicity of six rare serotype recombinant adenovirus vaccine vectors from subgroups B and D. J. Virol. 81, 4654-4663. doi: 10.1128/JVI.02696-06

Alexander, J., Ward, S., Mendy, J., Manayani, D. J., Farness, P., Avanzini, J. B., et al. (2012). Pre-clinical evaluation of a replication-competent recombinant adenovirus serotype 4 vaccine expressing influenza H5 hemagglutinin. PLoS One 7:e31177. doi: 10.1371/journal.pone.003 1177

Aoki, K., and Tagawa, Y. (2002). A twenty-one year surveillance of adenoviral conjunctivitis in Sapporo. Japan. Int. Ophthalmol. Clin. 42, 49-54. doi: 10.1097/ 00004397-200201000-00008

Aste-Amezaga, M., Bett, A. J., Wang, F., Casimiro, D. R., Antonello, J. M., Patel, D. K., et al. (2004). Quantitative adenovirus neutralization assays based on the secreted alkaline phosphatase reporter gene: application in epidemiologic studies and in the design of adenovector vaccines. Hum. Gene Ther. 15, 293-304. doi: 10.1089/104303404322886147

Barouch, D. H., Pau, M. G., Custers, J. H., Koudstaal, W., Kostense, S., Havenga, M. J., et al. (2004). Immunogenicity of recombinant adenovirus serotype 35 vaccine in the presence of pre-existing anti-Ad5 immunity. J. Immunol. 172, 6290-6297. doi: 10.4049/jimmunol.172.10.6290

Barraza, E. M., Ludwig, S. L., Gaydos, J. C., and Brundage, J. F. (1999). Reemergence of adenovirus type 4 acute respiratory disease in military trainees: report of an outbreak during a lapse in vaccination. J. Infect. Dis. 179, 1531-1533. doi: $10.1086 / 314772$

Berciaud, S., Rayne, F., Kassab, S., Jubert, C., Faure-Della Corte, M., Salin, F., et al. (2012). Adenovirus infections in Bordeaux University Hospital 2008-2010: clinical and virological features. J. Clin. Virol. 54, 302-307. doi: 10.1016/j.jcv. 2012.04.009

Choudhry, A., Mathena, J., Albano, J. D., Yacovone, M., and Collins, L. (2016). Safety evaluation of adenovirus type 4 and type 7 vaccine live, oral in military recruits. Vaccine 34, 4558-4564. doi: 10.1016/j.vaccine.2016.07.033

Clemmons, N. S., McCormic, Z. D., Gaydos, J. C., Hawksworth, A. W., and Jordan, N. N. (2017). Acute respiratory disease in US Army Trainees 3 years after reintroduction of adenovirus vaccine (1). Emerg. Infect. Dis. 23, 95-98. doi: 10.3201/eid2301.161297

Deal, C., Pekosz, A., and Ketner, G. (2013). Prospects for oral replicating adenovirus-vectored vaccines. Vaccine 31, 3236-3243. doi: 10.1016/j.vaccine. 2013.05.016

Dehghan, S., Seto, J., Liu, E. B., Walsh, M. P., Dyer, D. W., Chodosh, J., et al. (2013). Computational analysis of four human adenovirus type 4 genomes reveals

\section{FUNDING}

This study was supported by National Natural Science Foundation of China (31470892), National Key Research and Development Project (2016YFC1200900), National Science and Technology Major Project (2017ZX10204401003 and 2018ZX10101003005), the grant from Health and Family Planning Commission of Hunan Province (C2017085), Guangzhou Health Care and Cooperative Innovation Major Project (201803040004), and the grant of CAS Youth Innovation Promotion Association (2014328).

\section{SUPPLEMENTARY MATERIAL}

The Supplementary Material for this article can be found online at: https://www.frontiersin.org/articles/10.3389/fmicb. 2018.03040/full\#supplementary-material

molecular evolution through two interspecies recombination events. Virology 443, 197-207. doi: 10.1016/j.virol.2013.05.014

Dharmapuri, S., Peruzzi, D., and Aurisicchio, L. (2009). Engineered adenovirus serotypes for overcoming anti-vector immunity. Expert. Opin. Biol. Ther. 9, 1279-1287. doi: 10.1517/14712590903187053

Erdin, B. N., Pas, S. D., Durak, I., Schutten, M., and Sayiner, A. A. (2015). A 5-year study of adenoviruses causing conjunctivitis in Izmir. Turkey. J. Med. Virol. 87, 472-477. doi: 10.1002/jmv.24071

Erdman, D. D., Xu, W., Gerber, S. I., Gray, G. C., Schnurr, D., Kajon, A. E., et al. (2002). Molecular epidemiology of adenovirus type 7 in the United States, 1966-2000. Emerg. Infect. Dis. 8, 269-277. doi: 10.3201/eid0803.01 0190

Feng, Y., Sun, X., Ye, X., Feng, Y., Wang, J., Zheng, X., et al. (2018). Hexon and fiber of adenovirus type 14 and 55 are major targets of neutralizing antibody but only fiber-specific antibody contributes to cross-neutralizing activity. Virology 518, 272-283. doi: 10.1016/j.virol.2018.03.002

Ghebremedhin, B. (2014). Human adenovirus: viral pathogen with increasing importance. Eur. J. Microbiol. Immunol. 4, 26-33. doi: 10.1556/EuJMI.4.20 14.1.2

Hashimoto, S., Gonzalez, G., Harada, S., Oosako, H., Hanaoka, N., Hinokuma, R., et al. (2018). Recombinant type Human mastadenovirus D85 associated with epidemic keratoconjunctivitis since 2015 in Japan. J. Med. Virol. 90, 881-889. doi: 10.1002/jmv.25041

Hoke, C. H. Jr., and Snyder, C. E. Jr. (2013). History of the restoration of adenovirus type 4 and type 7 vaccine, live oral (Adenovirus Vaccine) in the context of the department of defense acquisition system. Vaccine 31, 1623-1632. doi: 10.1016/ j.vaccine.2012.12.029

Hong, J. Y., Lee, H. J., Piedra, P. A., Choi, E. H., Park, K. H., Koh, Y. Y., et al. (2001). Lower respiratory tract infections due to adenovirus in hospitalized Korean children: epidemiology, clinical features, and prognosis. Clin. Infect. Dis. 32, 1423-1429. doi: 10.1086/320146

Hsu, K. H. L., Lubeck, M. D., Davis, A. R., Bhat, R. A., Selling, B. H., Bhat, B. M., et al. (1992). Immunogenicity of recombinant adenovirus-respiratory syncytial virus-vaccines with adenovirus type-4. type-5, and type-7 vectors in dogs and a chimpanzee. J. Infect. Dis. 166, 769-775. doi: 10.1093/infdis/166.4.769

Hung, P. P., Chanda, P. K., Natuk, R. J., Mason, B. B., Chengalvala, M., Bhat, B. M., et al. (1990). Adenovirus vaccine strains genetically engineered to express Hiv-1 or Hbv antigens for use as live recombinant vaccines. Nat. Immun. Cell. Growth. Regul. 9, 160-164.

Kajan, G. L., Kajon, A. E., Pinto, A. C., Bartha, D., and Arnberg, N. (2017). The complete genome sequence of human adenovirus 84 , a highly recombinant new human mastadenovirus D type with a unique fiber gene. Virus Res. 242, 79-84. doi: 10.1016/j.virusres.2017.09.012 
Kajon, A. E., Moseley, J. M., Metzgar, D., Huong, H. S., Wadleigh, A., Ryan, M. A., et al. (2007). Molecular epidemiology of adenovirus type 4 infections in US military recruits in the postvaccination era (1997-2003). J. Infect. Dis. 196, 67-75. doi: 10.1086/518442

Khurana, S., Coyle, E. M., Manischewitz, J., King, L. R., Ishioka, G., Alexander, J., et al. (2015). Oral priming with replicating adenovirus serotype 4 followed by subunit $\mathrm{H} 5 \mathrm{~N} 1$ vaccine boost promotes antibody affinity maturation and expands H5N1 cross-clade neutralization. PLoS One 10:e0115476. doi: 10.1371/ journal.pone. 0115476

Kolavic-Gray, S. A., Binn, L. N., Sanchez, J. L., Cersovsky, S. B., Polyak, C. S., Mitchell-Raymundo, F., et al. (2002). Large epidemic of adenovirus type 4 infection among military trainees: epidemiological, clinical, and laboratory studies. Clin. Infect. Dis. 35, 808-818. doi: 10.1086/342573

Lanzi, A., Ben Youssef, G., Perricaudet, M., and Benihoud, K. (2011). Antiadenovirus humoral responses influence on the efficacy of vaccines based on epitope display on adenovirus capsid. Vaccine 29, 1463-1471. doi: 10.1016/j. vaccine.2010.12.025

Ludwig, S. L., Brundage, J. F., Kelley, P. W., Nang, R., Towle, C., Schnurr, D. P., et al. (1998). Prevalence of antibodies to adenovirus serotypes 4 and 7 among unimmunized US Army trainees: results of a retrospective nationwide seroprevalence survey. J. Infect. Dis. 178, 1776-1778. doi: 10.1086/31 4498

McNeill, K. M., Benton, F. R., Monteith, S. C., Tuchscherer, M. A., and Gaydos, J. C. (2000). Epidemic spread of adenovirus type 4-associated acute respiratory disease between US army installations. Emerg. Infect. Dis. 6, 415-419. doi: 10.3201/eid0604.000419

Peng, Y., Lai, M. M., Lou, Y. Y., Liu, Y. Q., Wang, H. Y., and Zheng, X. Q. (2016). Efficient induction of cross-presentating human B cell by transduction with human adenovirus type 7 vector. Immunol. Lett. 169, 41-51. doi: 10.1016/j. imlet.2015.11.011

Pichla-Gollon, S. L., Drinker, M., Zhou, X. Y., Xue, F., Rux, J. J., Gao, G. P., et al. (2007). Structure-based identification of a major neutralizing site in an adenovirus hexon. J. Virol. 81, 1680-1689. doi: 10.1128/Jvi.020 23-06

Radin, J. M., Hawksworth, A. W., Blair, P. J., Faix, D. J., Raman, R., Russell, K. L., et al. (2014). Dramatic decline of respiratory illness among US military recruits after the renewed use of adenovirus vaccines. Clin. Infect. Dis. 59, 962-968. doi: $10.1093 / \mathrm{cid} / \mathrm{ciu} 507$

Radke, J. R., and Cook, J. L. (2018). Human adenovirus infections: update and consideration of mechanisms of viral persistence. Curr. Opin. Infect. Dis. 31, 251-256. doi: 10.1097/Qco.0000000000000451

Scott, M. K., Chommanard, C., Lu, X., Appelgate, D., Grenz, L., Schneider, E., et al. (2016). Human adenovirus associated with severe respiratory infection. Oregon, USA, 2013-2014. Emerg. Infect. Dis. 22, 1044-1051. doi: 10.3201/ eid2206.151898

Sun, C., Zhang, Y., Feng, L., Pan, W., Zhang, M., Hong, Z., et al. (2011). Epidemiology of adenovirus type 5 neutralizing antibodies in healthy people and AIDS patients in Guangzhou, southern China. Vaccine 29, 3837-3841. doi: 10.1016/j.vaccine.2011.03.042

Tang, L., Wang, L., Tan, X., and Xu, W. (2011). Adenovirus serotype 7 associated with a severe lower respiratory tract disease outbreak in infants in Shaanxi province. China. Virol. J. 8:23. doi: 10.1186/1743-422X-8-23

Top, F. H. Jr. (1975). Control of adenovirus acute respiratory disease in U.S. Army trainees. J. Biol. Med. 48, 185-195.

Weaver, E. A. (2014). Vaccines within vaccines the use of adenovirus types 4 and 7 as influenza vaccine vectors. Hum. Vaccin. Immunother. 10, 544-556. doi: $10.4161 /$ hv. 27238

Yamamoto, D., Okamoto, M., Lupisan, S., Suzuki, A., Saito, M., Tamaki, R., et al. (2014). Impact of human adenovirus serotype 7 in hospitalized children with severe fatal pneumonia in the Philippines. Jpn. J. Infect. Dis. 67, 105-110. doi: 10.7883/yoken.67.105

Yang, W. X., Zou, X. H., Jiang, S. Y., Lu, N. N., Han, M., Zhao, J. H., et al. (2016). Prevalence of serum neutralizing antibodies to adenovirus type 5 (Ad5) and 41 (Ad41) in children is associated with age and sanitary conditions. Vaccine 34, 5579-5586. doi: 10.1016/j.vaccine.2016.09.043

Yang, X. X., Wang, Q. S., Liang, B. B., Wu, F. L., Li, H., Liu, H. B., et al. (2017). An outbreak of acute respiratory disease caused by a virus associated RNA II gene mutation strain of human adenovirus 7 in China, 2015. PLoS One 12:e0172519. doi: 10.1371/journal.pone.0172519

Yu, B., Zhou, Y., Wu, H., Wang, Z., Zhan, Y., Feng, X., et al. (2012). Seroprevalence of neutralizing antibodies to human adenovirus type 5 in healthy adults in China. J. Med. Virol. 84, 1408-1414. doi: 10.1002/jmv.23325

Yuan, X. H., Qu, Z. Y., Wu, X. M., Wang, Y. C., Liu, L., Wei, F. X., et al. (2009). Molecular modeling and epitopes mapping of human adenovirus type 3 hexon protein. Vaccine 27, 5103-5110. doi: 10.1016/j.vaccine.2009.06.041

Zhang, L., Zhao, N., Sha, J., Wang, C., Jin, X., Amer, S., et al. (2016). Virology and epidemiology analyses of global adenovirus-associated conjunctivitis outbreaks, 1953-2013. Epidemiol. Infect. 144, 1661-1672. doi: 10.1017/S095026881500 3246

Zheng, X., Rong, X., Feng, Y., Sun, X., Li, L., Wang, Q., et al. (2017). Seroprevalence of neutralizing antibodies against adenovirus type 14 and 55 in healthy adults in Southern China. Emerg. Microbes. Infect. 6:e43. doi: 10.1038/emi.2017.29

Conflict of Interest Statement: The authors declare that the research was conducted in the absence of any commercial or financial relationships that could be construed as a potential conflict of interest.

Copyright (c) 2018 Ye, Xiao, Zheng, Wang, Shu, Feng, Liu, Su, Wang, Li, Chen and Feng. This is an open-access article distributed under the terms of the Creative Commons Attribution License (CC BY). The use, distribution or reproduction in other forums is permitted, provided the original author(s) and the copyright owner(s) are credited and that the original publication in this journal is cited, in accordance with accepted academic practice. No use, distribution or reproduction is permitted which does not comply with these terms. 